\title{
Virtual Time Machine for Reproducible Network Emulation
}

\author{
Jin Chen \\ Beijing University of Posts and Telecommunications
}

Tao Huang

Beijing University of Posts and Telecommunications

Purple Mountain Laboratories

\author{
Jiang Liu \\ Beijing University of Posts and Telecommunications \\ Purple Mountain Laboratories \\ Jason Liu \\ Florida International University
}

\begin{abstract}
Reproducing network emulation experiments on diverse physical platforms with varying computation and communication resources is non-trivial. Many state-of-the-art network emulation testbeds do not guarantee timing fidelity. Consequently, results obtained from these testbeds can be misleading, especially when insufficient physical resources are provided to run the experiments. Reproducibility is far from being the norm. In this paper, we present a novel approach that can guarantee reproducible results for network emulation. Our system, called the Virtual Time Machine (VTM), takes advantage of both time dilation and carefully controlled scheduling of the virtual machines. Time dilation allows sufficiently scaled resources to run the experiments in virtual time, and controlled VM scheduling prescribes the precise timing of message passing for distributed applications - independent of the resource provisioning of the underlying physical testbed. Preliminary experiments show that VTM can guarantee reproducible results with varying time dilation, resource subscription, and VM scheduling scenarios.
\end{abstract}

\section{CCS CONCEPTS}

- Networks $\rightarrow$ Network experimentation; • Computing methodologies $\rightarrow$ Modeling methodologies; • Software and its engineering $\rightarrow$ Message passing.

\section{KEYWORDS}

Network emulation, network experiments, virtual machines, time dilation, reproducibility

\section{ACM Reference Format:}

Jin Chen, Jiang Liu, Tao Huang, and Jason Liu. 2019. Virtual Time Machine for Reproducible Network Emulation. In SIGSIM Principles of Advanced Discrete Simulation (SIGSIM-PADS '19), June 3-5, 2019, Chicago, IL, USA. ACM, New York, NY, USA, 10 pages. https://doi.org/10.1145/3316480.3322897

Permission to make digital or hard copies of all or part of this work for personal or classroom use is granted without fee provided that copies are not made or distributed for profit or commercial advantage and that copies bear this notice and the full citation on the first page. Copyrights for components of this work owned by others than ACM must be honored. Abstracting with credit is permitted. To copy otherwise, or republish, to post on servers or to redistribute to lists, requires prior specific permission and/or a fee. Request permissions from permissions@acm.org.

SIGSIM-PADS '19, June 3-5, 2019, Chicago, IL, USA

(C) 2019 Association for Computing Machinery.

ACM ISBN 978-1-4503-6723-3/19/06 . .\$15.00

https://doi.org/10.1145/3316480.3322897

\section{INTRODUCTION}

There has been increasing awareness in improving reproducibility in experimental computer science research. For example, ACM recently adopted a set of policies on artifact reviewing and badging for its publication procedures [1]. Many conferences have also embraced the idea of encouraging the authors to voluntarily turn in experiment artifacts along with their paper submissions, which include software, documentation, raw data, experiment scripts, and so on, in hope that they will be readily available with the published papers to allow other researchers to reproduce the results. For network research, it is important that researchers can run experiments using either the same or slightly different scenarios to obtain sufficiently similar results so that one can consistently draw conclusions about the functionality, capability, or performance of the target network design.

With the increasing presence of community-based network testbeds and shared compute infrastructures (such as GENI [7], NSFCloud [3, 4], and PAWR [22]), researchers are now capable of instantiating experiments on these testbeds with relative ease. However, having the ability to instantiate arbitrary experiments does not mean that all experiments conducted on these testbeds can be readily reproducible by themselves. On the contrary, given the complexity of today's network systems and the scale of the experiments, one may find it rather difficult to acquire the same computing resources and network conditions in order to produce and reproduce desirable experiment outcome.

For this, network emulation offers a good compromise. Network emulation allows one to set up experiments using operating system facilities, such as virtual machines, software switches and routers, and programmable packet forwarding mechanisms (e.g., virtual Ethernet pairs, IP chains, and network tunneling interfaces). An emulated network can operate in real time, support arbitrary network connectivity through virtualization, and perform necessary traffic shaping by injecting artificial delays in the network transmission.

A case in point is Mininet [11, 15], which is a popular container-based emulation environment built on Linux to test OpenFlow applications [19]. Mininet uses lightweight OS containers to emulate individual network hosts. Each virtual host corresponds to a container attached to a separate network namespace. Each container is facilitated with virtual network interfaces configured with distinct IP addresses and independent functions of the TCP/IP stack. The containers can be virtually connected through kernel routing and 
forwarding. The virtual network interfaces of the hosts can be either connected with one another or with instances of the Open vSwitch (OVS) [20], a production-quality software switch augmented with OpenFlow capabilities supporting Software Defined Network (SDN) applications.

Mininet experiments consist of a set of interconnected virtual hosts and virtual switches organized in an arbitrary network topology. Real applications can run directly on the virtual hosts. Mininet provides performance limiting and isolation features, using existing Linux tools, such as cgroups for scheduling and CPU resource management, and tc to control the network link properties. Consequently, each virtual host in Mininet can be configured to consume only a fraction of the overall system's CPU resources. Each network link can be configured with specific bandwidth, latency, and packet loss probability. To enable OpenFlow, one can connect an OpenFlow controller with the OVS software switches for a full-fledged software-defined network experimentation.

Using virtual machines ${ }^{1}$ and software routers allows resource over-subscription of the network experiments. That is, a researcher can instantiate a virtual network consisted of many virtual hosts and routers on just a few physical machines. In doing so, one can effectively create the system prototypes and conduct large-scale experiments with only limited resources. Unfortunately, resource over-subscription also has its limitations. The network configuration (such as the link bandwidth) must be constrained according to the physical capacity of the underlying testbed. Packet forwarding on the virtual network consumes CPU and memory resources. In Mininet, for example, the aggregate capacity in emulation cannot be more than a few gigabits per second (the exact value depends on the processing capability of the underlying physical machine). For network-bound experiments (with high network traffic intensity), this can result in a significant limitation. Similarly, for CPU-bound experiments, one must carefully allocate the CPU resources to the virtual hosts. For large experiments with many virtual machines, the actual CPU resources assigned to each virtual machine would be severely limited due to resource over-subscription. As a result, the timing accuracy cannot be guaranteed. We know for fact that Mininet's performance measurement can be extremely unreliable for large network experiments with heavy workload.

All these factors can substantially affect the reproducibility of network emulation. The fundamental question here is whether we can conduct network emulation experiments and maintain fidelity of the results independent of the underlying physical resources, particularly in resource oversubscription situations. For this, we propose Virtual Time Machine (VTM). The main objective is to enable reproducible network experiments on arbitrary compute resources with varying degree of resource subscription. VTM can support network experiments - some with a large number of virtual hosts and routers, and also with intensive workload and traffic

\footnotetext{
${ }^{1}$ In this paper, we do not differentiate virtual machines and containers as they both provide an isolated environment for running the test network applications for experimentation purposes.
}

demand - on various physical platforms (e.g., with different number of compute nodes, different processing capabilities, different interconnection bandwidth and latency, etc.), and yet still produce the same results.

To allow varying degree of source subscription levels for running large-scale network emulation and enabling network experiments with high capacity links, we apply time dilation. Time dilation can change a virtual machine's notion of how time progresses when running the applications [10]. This can be achieved by enlarging the interval between timer interrupts delivered to the virtual machines by a specified scaling factor, called the Time Dilation Factor (TDF). Time dilation can scale the perceived $\mathrm{I} / \mathrm{O}$ rate and the processing power on the virtual machines by the same factor. For instance, if a virtual machine has a TDF of ten, it means that the time, as perceived by applications running on the virtual machine, will advance at a pace ten times slower than the wall-clock time. As such, the applications can experience a tenfold increase in both network and CPU processing capacities. To allow reproducibility, we incorporate the time dilation technique with the scheduling of the virtual machines so that we can control the exact timing of the network transactions (sending and receiving message) regardless of the resource subscription level (i.e., how many virtual machines are assigned to run on a physical machine).

Our VTM implementation extends TimeKeeper [14], which allows dynamic TDF adjustment based on the resource utilization of the emulated experiment, and supports VM scheduling based on virtual time advancement. We combine time dilation, virtual machine scheduling, and traffic shaping capabilities in such a way that packet processing and delivery can be guaranteed to adhere to the bandwidth and latency constraints of the target network at different resource subscription levels. For implementation, we construct an emulated network with a set of virtual hosts, routers, and links, instantiated on one or multiple physical machines interconnected by a network. Each virtual host is implemented as a Linux container. Each virtual router/switch is implemented as an OVS instance running inside a container. The containers are assigned potentially with different time dilation factors depending on the network configuration and workload. All network links are set with respective bandwidth and latency limitations using Linux tc and netem. The time dilation factors (TDFs) and the associated tc/netem parameters can be determined as a function of the processing capability and the networking speed of underlying physical platform, relative to the demand of the target emulation scenario. The virtual machines at each physical machine are scheduled to run deterministically in a round-robin fashion. At each round, each virtual machine is allowed to run only for a specific duration determined by the virtual machine's TDF. To realize the specific delays of messages sent between the virtual machines (regardless whether they reside on the same physical machine or not), we can calculate the real time delay based on the runtime schedule of the two virtual machines. In doing so, we can remove the interference caused by competing virtual machines simultaneously running on the physical machine. In 
other words, we allow the network experiment to achieve reproducible results independent from the testbed.

The rest of this paper is organized as follows. In Section 2, we discuss related work. We describe our method in more detail in Section 3. Furthermore, we discuss implementation issues in Section 4. We conducted preliminary experiments to validate our method; the results are described in Section 5 . Finally, we summarize the paper and outline future directions in Section 6.

\section{RELATED WORK}

Incorporating virtual time for studying real systems has been attempted in various ways. Direct execution-driven simulation incorporates network functions and protocol stack from real systems into discrete-event simulation, e.g., [5, 16-18, 23, 24].

As virtual machines are now oftentimes used for network emulation, integrating virtual time control directly in the virtual machine system has become quite common. The original idea of dilating time and integrating virtual time control in real systems was first introduced by Gupta et al. [10]. By slowing down the progression of time perceived by applications through modification of timing related kernel functions, one can conduct emulation experiments with higher CPU and I/O capabilities than what could be provided by the underlying physical testbed. In a different way, Bergstrom et al. [2] proposed a virtual time management system, which applies binary executable modification to the network stack of the operating systems to allow dynamic dilation and contraction of time for improving resource utilization during experiments.

Gupta et al. [9] applied the idea of time dilation to scale the perceived resource capacity of various hardware components of network systems for performance studies. Grau et al. [8] proposed a conservative synchronization mechanism to regulate the progression of virtual time across virtual machines in network emulation. Erazo et al. [6] proposed an emulation system for TCP performance evaluation. By controlling the virtual time advancement synchronously both in simulation and on the virtual machines, the system is capable of studying large-scale network performance with desirable accuracy. In a similar fashion, Weingärtner et al. [25] also combined simulation and virtual machines with virtual time control for large-scale network experiments. In addition, they also proposed synchronization algorithms to allow time coordinated execution of network simulation and virtual machines.

The virtual time systems mentioned above were all implemented on Xen. Zheng and Nicol [28] created an implementation of time dilation on OpenVZ. Jin et al. [27] later extended the system to fully integrate with a parallel discrete-event simulation framework called S3F. In doing so, the system is able to conduct experiments with native Linux applications that run inside the OpenVZ containers and operate synchronously in virtual time with parallel simulation.

Yan and Jin [26] implemented the time dilation mechanism for lightweight Linux containers used in Mininet. Their system, Mininet-VT, provides better time control ability where the containers are synchronized in virtual time via VM scheduling. It also allows dynamic adjustment of the time dilation factor of the virtual machines based on the resource utilization of the emulated experiment. Lamps et al. [13, 14] created a separate implementation of the time dilation system using Linux containers. Their system, TimeKeeper, also provides fine-grained control over VM scheduling and allows containers to have different and dynamically changing time dilation factors. The system can be easily installed as a kernel modification and a kernel module, and can be readily integrated with different simulators and emulators. Our system is built on TimeKeeper.

Our approach is distinct from the previous methods in two important aspects. First, our goal is different. We focus on enabling reproducibility of emulation experiments. For that, we need to deal with issues beyond the simple control of advancing virtual time and maintaining synchronization of virtual machines. Second, we coordinate time dilation and VM scheduling in order to perform time accurate network operations so that the emulated network experiments are able to produce similar results independent from the physical capacity of the underlying testbeds.

\section{VIRTUAL TIME MACHINE}

In this section, we describe the design of VTM. In particular, we describe how a virtual network is mapped and realized on a physical testbed with VTM support, how to determine the time dilation for the virtual machines, and how to schedule the virtual machines and perform network operations in concert with the computation and communication demand of the experiment and the available resources of the physical testbed.

\subsection{Target Network and Physical Testbed}

The target network in an experiment can be represented as a directed graph $G=(V, E)$, where $V=\left\{v_{1}, v_{2}, \cdots, v_{n}\right\}$ is the set of nodes (including hosts, switches, and routers), and $E=\left\{e_{1}, e_{2}, \cdots, e_{m}\right\}$ is the set of (directed) links between the nodes. The graph consists of $n$ nodes and $m$ links. The target network will be emulated using a set of virtual machines (containers in our implementation). The software routers/switches are placed inside the virtual machines. So are the test applications and network protocols. The network connections between the virtual machines are pre-configured with specific bandwidth and delay constraints according to the target network specification.

We use $\pi_{i}$ to denote the minimum processing speed of node $v_{i} \in V$. For simplicity, we normalize this processing speed in units of $\mathrm{GHz}$. Here, we assume that applications will not alter their runtime behavior and therefore shall be able to generate reproducible results when running on the virtual machines guaranteed with the minimum amount of CPU cycles ${ }^{2}$. The value $\pi_{i}$ is used to determine the time

\footnotetext{
${ }^{2} \mathrm{GHz}$ and Gbps are normalized units for describing the minimal requirement of the physical machine's capacity for performing reproducible network emulation studies.
} 
dilation factor of the corresponding virtual machine; in other words, it indicates the minimum amount of CPU resources needed to run the target applications without creating timing distortions. For most I/O-driven applications, however, this value may not be as important, since the application behavior would be determined by I/O events. In such cases, this value can be set to zero, which means that the CPU processing speed would be regarded as negligible when compared to the network processing speed, which we define below.

The target network will be emulated on a testbed with $M$ physical machines, so that each physical machine will run a set of virtual machines representing a subset of the target network. Ideally, the virtual machines are evenly distributed across the physical machines so that the load is balanced among them and the communication across the physical network is minimized. To a certain extent, load balancing and traffic optimization can be approximated and determined beforehand using graph partitioning tools, such as METIS [12]. Here, we assume that the user simply specifies the network partitions. Suppose the target network is divided into a set of $M$ partitions: $\left\{P_{1}, P_{2}, \cdots, P_{M}\right\}$, where $P_{k}$ represents the set of nodes belonging to the $k^{t h}$ partition. We have:

$$
\bigcup_{1 \leq k \leq M} P_{k}=V \text { and } \bigcap_{1 \leq k \leq M} P_{k}=\phi
$$

Further, let $\Pi_{k}$ be the CPU processing speed of the $k^{t h}$ physical machine (again in units of $\mathrm{GHz}$ ). Let $\Theta_{k}$ be the network processing speed in terms of packet handling rate on the same physical machine (in units of Gbps). Although our framework can handle heterogeneous computing environments, for easier exposition, in the sections to follow, we assume that the physical testbed consists of only homogenous machines. That is, $\Pi=\Pi_{k}$ and $\Theta=\Theta_{k}$ for $0 \leq k<M$. Let $\Omega$ be the network bandwidth connecting the physical machines (in Gbps). As we show momentarily, the minimum processing speed $\pi_{i}$ of node $v_{i}$, together with the CPU processing speed $\Pi$ and the network processing speed $\Theta$ of the physical machine will be used to determine the time dilation factor and the scheduling of the virtual machines.

\subsection{Determining Time Dilation}

Each node in the target network is instantiated as a separate virtual machine. (In our implementation, we use Linux containers.) Each switch/router in the target network is implemented as an OVS instance running inside a separate virtual machine. For node $v_{i} \in V$, let $\lambda_{i}$ be the time dilation factor (TDF). In this section, we discuss how to determine the TDFs for the all nodes in the target network so that the network experiment can be realized with accuracy using the available resources on the physical testbed.

For link $e=\left(v_{i}, v_{j}\right) \in E$ from node $v_{i}$ to $v_{j}$, we use $b_{i j}$ and $d_{i j}$ to denote the bandwidth and latency, respectively. Note that, in many cases, the links are bidirectional; that is, $b_{i j}=b_{j i}$ and $d_{i j}=d_{j i}$. However, they do not generally have to be bidirectional. Also note that both $b_{i j}$ and $d_{i j}$ are specified for the target network. With time dilation, the actually bandwidth and latency will be different. Let $\omega_{i j}$ and $\delta_{i j}$ be the actual bandwidth and latency in real time on the physical system. The relationship between the TDF of a node, the emulated and actual bandwidth of a link emitted from the node can be expressed as follows:

$$
\omega_{i j}=\frac{b_{i j}}{\lambda_{i}}, \forall\left(v_{i}, v_{j}\right) \in E,
$$

Time dilation can scale both the processing speed and the perceived I/O rate at the virtual machine by the same factor. The actual latency of the link on the physical testbed has to be determined with respect to the scheduling and synchronization of the virtual machines on the physical testbed. We discuss this in the next section.

For now, it is sufficient for us to determine the TDFs for all nodes, by considering three constraints, namely, NPC, PPC, and NTC.

1) Nodal Processing Condition (NPC) stipulates that the required processing speed of each node must be satisfied on the time dilated machine:

$$
\sum_{v_{i} \in P_{k}} \frac{\pi_{i}}{\lambda_{i}} \leq \Pi, \text { where } 0 \leq k<M
$$

2) Packet Processing Condition (PPC) stipulates that the maximum rate of packet handling at a physical machine cannot exceed the machine's network processing speed:

$$
\sum_{\substack{\left(v_{i}, v_{j}\right) \in E \wedge \\\left(v_{i} \in P_{k} \vee v_{j} \in P_{k}\right)}} \omega_{i j}=\sum_{\substack{\left(v_{i}, v_{j}\right) \in E \wedge \\\left(v_{i} \in P_{k} \vee v_{j} \in P_{k}\right)}} \frac{b_{i j}}{\lambda_{i}} \leq \Theta
$$

where $0 \leq k<M$. The maximum rate of packet handling on a physical machine (in units of $\mathrm{GHz}$ ) is the sum of bandwidth of all links either starting from or ending at a node assigned to the physical machine, or both.

3) Network Traffic Condition (NTC) stipulates that traffic generated by a physical machine to another cannot exceed the bandwidth of the physical network connecting the machines:

$$
\sum_{\substack{\left(v_{i}, v_{j}\right) \in E \wedge \\ v_{i} \in P_{k} \wedge v_{j} \notin P_{k}}} \omega_{i j}=\sum_{\substack{\left(v_{i}, v_{j}\right) \in E \wedge \\ v_{i} \in P_{k} \wedge v_{j} \notin P_{k}}} \frac{b_{i j}}{\lambda_{i}} \leq \Omega
$$

where $0 \leq k<M$. The left side of the inequality is the maximum network traffic that can be sent from physical machine $k$ to all other physical machines.

We can formulate a simple optimization problem to determine the TDFs of all nodes in the target network:

$$
\begin{gathered}
\text { Minimize: } \sum_{v_{i} \in V} \lambda_{i} \\
\text { Subject to: (1), (2), and (3). }
\end{gathered}
$$

We want to minimize time dilation since a larger time dilation factor would entail a longer execution time for the experiment. We have also observed that too large a time dilation factor may actually introduce undesirable artifacts in the system that would negatively affect the overall accuracy of the emulation experiment. In practice, we can approximate the solution by choosing the TDF $\lambda_{i}$ for node $v_{i}$ to be in proportion to the relative computing and networking demand 
of the node, as defined in the following:

$$
\begin{gathered}
\phi_{i}=\max \left\{\frac{\pi_{i}}{\Pi}, \frac{1}{\Theta}\left(\sum_{\left(v_{i}, v_{j}\right) \in E} b_{i j}+\sum_{\left(v_{j}, v_{i}\right) \in E} b_{j i}\right),\right. \\
\left.\frac{1}{\Omega} \sum_{\substack{\left(v_{i}, v_{j}\right) \in E \wedge \\
v_{i} \in P_{k} \wedge v_{j} \notin P_{k}}} b_{i j}\right\}
\end{gathered}
$$

The first term on the right side of the equation represents the relative CPU processing demand, the second term is the relative packet handling demand, and the last term is the relative network traffic demand. The overall demand of the node can be then defined as the largest of the three and we set the time dilation factor $\lambda_{i}$ to be proportional to this value; that is, $\lambda_{i}=c \cdot \phi_{i}$, where $c$ is the constant proportion factor. We can calculate $\phi_{i}$ for all nodes, and then replace $\lambda_{i}$ with $\phi_{i}$ and $c$ in equations (1), (2), and (3) to solve for the smallest $c$.

\subsection{Determining Link Delays}

Even with ample time dilation to allow sufficient support for computation and communication, large network experiments may not be reproducible when multiple virtual machines are allocated and run at each physical machine. This is because the exact time a virtual machine sends or receives a message depends on the time at which the virtual machine is scheduled to run. VM scheduling in turn depends on the resource subscription level. For instance, if each virtual machine is given a time slice of $1 \mathrm{~ms}$ to run at a time, and if there are ten virtual machines to emulate on a physical machine, the communication delay between the virtual machines can vary from 0 to $9 \mathrm{~ms}$. If we have hundreds of virtual machines on each physical machine, which may not be uncommon for large experiments, the delay range can be quite significant. If uncontrolled, this would contribute to the timing uncertainties of the emulation experiment.

To allow reproducibility, we need to control the network delays with respect to the resource subscription level and in accordance with the time dilation and scheduling of the virtual machines. In VTM, we adopt a fixed scheduling policy so that we can calculate the exact real-time delay for each message being sent between the virtual machines. More specifically, we schedule the virtual machines assigned to a physical machine in a round-robin fashion. Suppose a given physical machine $k$ has $q(k)$ nodes: $v_{1}, v_{2}, \cdots, v_{q(k)}$. Without loss of generality, suppose we schedule them in the natural order; that is, $v_{1}$ before $v_{2}$ before $v_{3}$, and so on. Each time a virtual machine is scheduled to run, the VM scheduler will let it run for a fixed amount of virtual time, which we call the virtual time slice denoted as $\tau$. If the virtual machine for node $v_{i}$ has a time dilation factor of $\lambda_{i}$, we run the virtual machine for a duration of $\tau \lambda_{i}$ in real time at each round.

To choose the right size for the virtual time slice, on the one hand, we need to make sure that the virtual machines can run long enough to compensate for the overhead for scheduling them. On the other hand, the virtual time slice should be no larger than the latency of the network links striding across the virtual machines. Otherwise, a message sent from one virtual machine to another may arrive at its destination within the same round. This would cause causality errors since the virtual machine receiving the message may have already been scheduled before the virtual machine sending the message, and therefore have its virtual time already advanced beyond the designated receive time. This is somewhat similar to the concept of lookahead in parallel discrete-event simulation. In summary, to avoid causality issues, we must guarantee that:

$$
\tau \leq \min _{\left(v_{i}, v_{j}\right) \in E} d_{i j}
$$

We name the total real time to run the virtual machines in one round among all physical machines as an epoch, denoted by $\Delta$. That is,

$$
\Delta=\max _{0 \leq k<M} \sum_{1 \leq i \leq q(k)} \tau \lambda_{i}
$$

For the virtual machine representing node $v_{i}$, let $l\left(v_{i}\right)$ be the lead time, which is defined as the wall-clock time from the beginning of the current epoch to the time when the virtual machine is run; and let $r\left(v_{i}\right)$ be the trail time, which is defined as the wall-clock time from the end of the time slice of $v_{i}$ to the end of the current epoch. Because of the fixed round-robin VM scheduling, the lead time and the trail time for all virtual machines should remain constant between different rounds:

$$
\begin{aligned}
& l\left(v_{i}\right)=\sum_{0 \leq k<i} \tau \lambda_{i} \\
& r\left(v_{i}\right)=\Delta-\sum_{0 \leq k \leq i} \tau \lambda_{i}
\end{aligned}
$$

Note that $\Delta=l\left(v_{i}\right)+\tau \lambda_{i}+r\left(v_{i}\right)$.

Fig. 1 shows the scheduling of the virtual machines both in real and virtual time. We show two virtual machines $V M_{i}$ and $V M_{j}$ within each round. The $\mathrm{x}$-axis is the real time and the $\mathrm{y}$-axis is the virtual time. The virtual machines are scheduled in a round-robin fashion, and at each round, each virtual machine runs and advances its virtual time by a fixed amount that equals the virtual time slice $\tau$. The figure also shows the delivery of a message from virtual machine $V M_{i}$ to virtual machine $V M_{j}$. The link delay from $V M_{i}$ to $V M_{j}$ is $d_{i j}$ in virtual time. The problem at hand is how to calculate the real-time delay $t_{i j}$ so that we can precisely deliver the message in emulation so that it can be reproduced for different runs, on different experiment platforms, independent of the VM scheduling ordering.

To calculate $t_{i j}$, we need to make a few more definitions. Let $x_{1}$ and $y_{1}$ be the virtual and wall-clock time during $V M_{i}$ 's time slice when the message is sent. Similarly, let $x_{2}$ and $y_{2}$ be the virtual and wall-clock time during $V M_{j}$ 's time slice when the message is received (at least one round after). Fig. 2 illustrates the sending and receiving of the message. Assume that in the time dilated system the virtual time and the real time progresses linearly according to the time dilation factor 


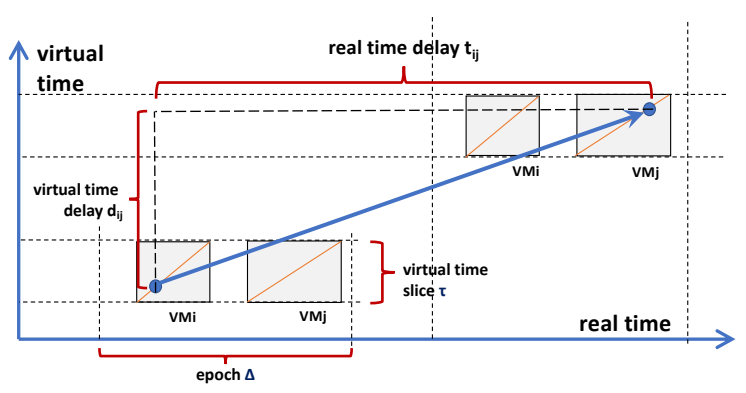

Figure 1: VM scheduling and delay calculation.

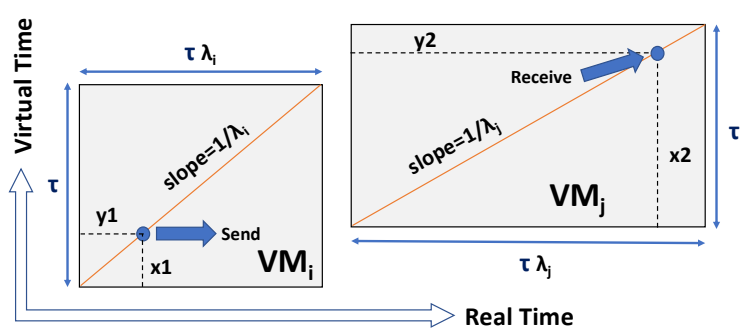

Figure 2: The sending and receiving of a message.

of the virtual machine ${ }^{3}$. In other words, time moves along the diagonal line of the rectangle as indicated in Fig. 2. The slope of the diagonal line is inversely proportional to the time dilation factor of the virtual machine.

Let $t$ be the wall-clock time at which the message is sent from $V M_{i}$. Let $s$ be the starting wall-clock time of the current round (which can be obtained easily by recording the time when each round starts during experiment). Recall $l\left(v_{i}\right)$ and $r\left(v_{i}\right)$ denote the lead time and the trail time of virtual machine $V M_{i}$ as defined in Eqn (7) and (8), respectively. We first calculate $y_{1}, x_{1}, x_{2}$, and $y_{2}$ in order:

$$
\begin{aligned}
y_{1} & =t-s-l\left(v_{i}\right) \\
x_{1} & =y_{1} / \lambda_{i}=\frac{t-s-l\left(v_{i}\right)}{\lambda_{i}} \\
x_{2} & =\left(x_{1}+d_{i j}\right) \bmod \tau \\
& =\left(\frac{t-s-l\left(v_{i}\right)}{\lambda_{i}}+d_{i j}\right) \bmod \tau \\
y_{2} & =\lambda_{j} x_{2} \\
& =\lambda_{j}\left[\left(\frac{t-s-l\left(v_{i}\right)}{\lambda_{i}}+d_{i j}\right) \bmod \tau\right]
\end{aligned}
$$

Then we calculate the number of rounds between the round in which the message is sent and the round in which the message is received:

$$
a=\left\lfloor\frac{x_{1}+d_{i j}}{\tau}\right\rfloor-\left\lfloor\frac{x_{1}}{\tau}\right\rfloor-1
$$

\footnotetext{
${ }^{3}$ Time dilation is applied only when the application makes time related system calls, such as sleep or gettimeofday. It is an approximation that the time is passing smoothly in this fashion.
}

And finally we derive the real-time delay:

$$
t_{i j}=\left(\tau \lambda_{i}-y_{1}+r\left(v_{i}\right)\right)+a \Delta+\left(l\left(v_{j}\right)+y_{2}\right)
$$

The right-hand side of the equation consists of three terms: the first term is the remaining real time between when the message is sent and the end of the current epoch; the second term is the number of epochs in-between the round in which the message is sent and the round in which the message is received; and the third term is the real time between the beginning of the epoch and when the message is received. Note that all three terms depend on $t$ (or $y_{1}$ in that matter), which is the exact wall-clock time (within the time slice) when the message is sent from the virtual machine.

We see two major problems related to calculating the real-time delay of individual messages based on their send time. First, there could be significant overhead for calculating and scheduling the arrival time of individual messages. This overhead cannot be underestimated as it can cause substantial slowdown of the emulation experiment if not handled with care. Second, we also need to consider the queuing effect as the messages compete for the bandwidth. The delay calculation and message scheduling need to be compatible with the queue management in the operating system. As far as we know, there is no existing system facilities that would allow us to easily incorporate the proposed per-message delay operations. We need to simplify the real-time delay calculations. For the rest of this section, we describe a simple approximation method.

If we assume that the network delay $d_{i j}$ for all links in the target network is divisible by the chosen time slice $\tau$, we can significantly simplify the calculations above. First, we will have $x_{1}=x_{2}$, and thus $y_{1}=x_{1} \lambda_{i}$, and $y_{2}=x_{1} \lambda_{j}$. Second, the number of rounds in-between can be simplified as $a=d_{i j} / \tau-1$. Finally, the real-time delay can be expressed as follows:

$$
\begin{aligned}
t_{i j} & =\left(r\left(v_{i}\right)+l\left(v_{j}\right)+a \Delta\right)+\left(\tau \lambda_{i}+y_{2}-y_{1}\right) \\
& =\left(r\left(v_{i}\right)+l\left(v_{j}\right)+\frac{d_{i j}-\tau}{\tau} \Delta\right)+\left(\left(\tau-x_{1}\right) \lambda_{i}+x_{1} \lambda_{j}\right)
\end{aligned}
$$

Note that the right-hand side of Eqn (15) has been consolidated into two terms. The first term, $r\left(v_{i}\right)+l\left(v_{j}\right)+\left(d_{i j}-\right.$ $\tau) \Delta / \tau$, is a fixed value for the experiment, as it depends merely on the virtual time delay $d_{i j}$, the size of the virtual time slice, the size of the epoch, and the fixed scheduling positions of the sending and receiving virtual machines within each round. The second term, $\left(\tau-x_{1}\right) \lambda_{i}+x_{1} \lambda_{j}$, still depends on $x_{1}$. However, we can see that if $x_{1}=0$, the value of the second term is $\tau \lambda_{i}$; and if $x_{1}=\tau$, it is $\tau \lambda_{j}$. To simplify message handling, we can approximate the second term by using an average of these two values, which is a fixed value for the experiment:

$$
t_{i j}=r\left(v_{i}\right)+l\left(v_{j}\right)+\frac{d_{i j}-\tau}{\tau} \Delta+\frac{\tau}{2}\left(\lambda_{i}+\lambda_{j}\right)
$$

In the next section, we describe the method which we use to implement the bandwidth and delay constraints using 
existing operating system facilities to achieve reproducible results.

\section{IMPLEMENTATION}

We developed a prototype that uses Linux containers (LXC) to implement the nodes in the target network. We use OVS [20] to represent routers and switches, and we place user-level OVS instances within the containers so that they can achieve the desired time dilation. For network links, we use virtual Ethernet (veth) pairs to connect the hosts with the switches and routers. The veth devices are always created in pairs and act as tunnels between network namespaces, where both end-points function as Ethernet devices within the containers.

We modified the TimeKeeper [14] for time dilation, which is a Linux kernel patch with the ability to assign different time dilation factors for containers. It has also been integrated with Mininet [15] for easy experiment setup. TimeKeeper comes with the ability to schedule containers by providing functions to freeze (i.e., stop executing) and unfreeze (i.e., resume executing) the containers. When unfrozen, a container may run and advance its virtual time by a fixed amount (the virtual time slice in our case). We use the TimeKeeper facilities together with a high-resolution timer (in Python) to implement the round-robin VM scheduling described in the previous section.

We use Linux Traffic Control (tc), in particular, the Token Bucket Filter (TBF) and the Hierarchy Token Bucket (HTB), for bandwidth sharing and traffic limiting, and the Network Emulator (netem) for facilitating message delays. tc applies specific queuing disciplines (qdisc) to manipulate traffic: Whenever the kernel needs to send a packet to a network interface, the packet is enqueued in the qdisc configured for that interface. TBF and HTB are specific queuing disciplines for traffic shaping. netem is an enhancement of tc, which is built on existing Quality of Service (QoS) and Differentiated Services (diffserv) facilities in the Linux kernel.

To emulate a link from virtual machine $V M_{i}$ to virtual machine $V M_{j}$ in the target network with bandwidth $b_{i j}$ and delay $d_{i j}$, our implementation follows two steps: In the first step, we create a virtual Ethernet pair to connect the senderside container with a Linux bridge. At one end of the veth pair is the virtual network device within the container and we attach it to the main container process so that it operates in virtual time. We use netem on the virtual device and set the latency to be as follows:

$$
t_{i j}^{V}=d_{i j}-\tau
$$

The real-time delay corresponding to $t_{i j}^{V}$ is $\left(\frac{d_{i j}-\tau}{\tau} \Delta\right)$. We also add an HTB/TBF queuing discipline and set the bandwidth to be $b_{i j}$. Note that the delay and bandwidth limit will apply to all outgoing packets from the device in virtual time.

In the second step, we create another virtual Ethernet pair to connect the bridge to the receive-side container. We also use netem on the virtual device attached to the bridge to apply additional real-time delay to packets as needed, so that the packets will get to the destination container at

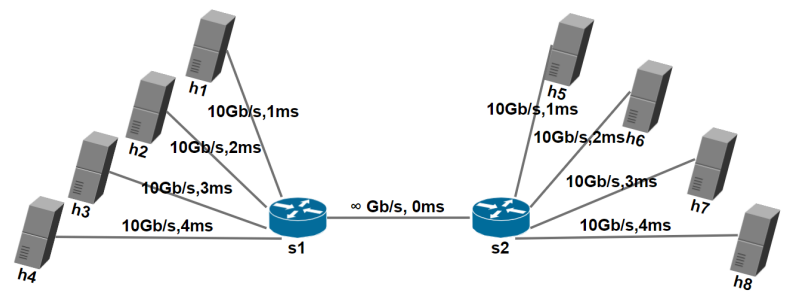

Figure 3: A dumbbell topology.

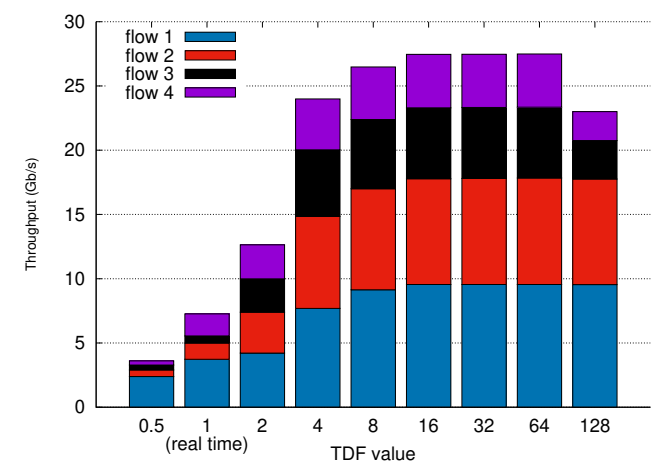

Figure 4: TCP throughput with varying time dilation.

the expected arrival time. The additional delay needs to compensate for the differences in time at which the VMs are scheduled to run. More specifically, we set the real-time delay to be:

$$
t_{i j}^{R}=r\left(v_{i}\right)+l\left(v_{j}\right)+\frac{\tau}{2}\left(\lambda_{i}+\lambda_{j}\right)
$$

Note that combining the virtual time delay $\left(t_{i j}^{V}\right.$ in step 1$)$ and the real-time delay $\left(t_{i j}^{R}\right.$ in step 2$)$ will result in exactly the same delay as specified in Eqn (16).

\section{EXPERIMENTS}

We conducted preliminary experiments to validate our prototype VTM system. All experiments reported in this section were run on the same workstation, which is a Dell PowerEdge R730 Server equipped with eight Intel ${ }^{\circledR}$ Xeon ${ }^{\circledR}$ E5-2609 v3 $1.90 \mathrm{GHz}$ processors and 32GB RAM. The system runs 64-bit Ubuntu 14.04 OS upgraded to kernel 3.13.1. We installed TimeKeeper and Mininet 2.3.0d4.

We performed three set of experiments to evaluate the system's ability to reproduce results. In all experiments, we set the virtual time slice of the round-robin VM scheduling to be $1 \mathrm{~ms}$. All experiments were run on a single physical machine. We defer distributed emulation experiments to future work.

\subsection{Different Time Dilation}

In the first set of experiments, we aim to evaluate the system's ability to reproduce results with different time dilation. For this, we used a dumbbell shaped network topology as 
shown in Fig. 3. There were two routers $\left(s_{1}\right.$ and $\left.s_{2}\right)$ in the center connected with a large-bandwidth low-latency link (we simply set infinite capacity and zero latency for the setup). On either side of the dumbbell, we connected four hosts to the router. The connections between the host and the router were configured with $10 \mathrm{~Gb} / \mathrm{s}$ bandwidth and different latencies from $1 \mathrm{~ms}$ to $4 \mathrm{~ms}$. We directed four TCP flows using iperf, one from a host on one side of the dumbbell to the corresponding host on the other side of the dumbbell. The round-trip time of the four flows is thus ranged from $4 \mathrm{~ms}$ to $16 \mathrm{~ms}$, and the aggregate throughput could stack up to $40 \mathrm{~Gb} / \mathrm{s}$.

In this experiment, we varied the time dilation factor of the containers from 0.5 to 128 doubling each time. All the ten containers in this experiment adopted the same time dilation factor. Fig. 4 is a stacked bar-chart that shows the throughput achieved by the four TCP flows under different TDF values. We can make two observations. First, the aggregate throughput drops significantly for TDF values below 8 . This is expected. The experiment is not reproducible due to insufficient resource subscription. Second, when TDF is too large (128 in our experiment), the result also shows lower aggregate throughput, which slightly deviates from the expected. We speculate that large time dilation might have amplified the artifacts in the system and contributed to the errors. This may be due to the inaccuracies in the timer implementation. Furthermore, time dilation is an approximation: the system is only able to adjust its (virtual time) clock when timing related system calls are invoked. We have observed that the errors appear to be more apparent for flows over high-delay and high-bandwidth links (such as flow 4 ), where the buffer size can vary significantly due to the non-time-dilated protocol actions causing the reduced throughput.

\subsection{Different Resource Subscription}

In the second set of experiments, we aim to evaluate the system's ability to reproduce results under different resource subscription levels. At the time of the experiments, we were limited by the choice of available physical platforms capable of running VTM. To achieve different resource subscription levels, we resorted to use the CPULimitedHost feature in Mininet to perform CPU scaling. Mininet uses Linux control group (cgroup) to set the CPU usage limit for the containers. Using this tool, we could effectively change the resource subscription level for the network experiments.

We again used the dumbbell topology and created four TCP flows using iperf as in the previous experiment. Different from the previous setup, we assigned the same bandwidth $(1 \mathrm{~Gb} / \mathrm{s})$ and delay $(1 \mathrm{~ms})$ for all links connecting the hosts with the routers. We also created a bottleneck link between the two routers in the center, and used tc filter so that each flow was limited to $20 \mathrm{Mb} / \mathrm{s}$ bandwidth and $30 \mathrm{~ms}$ delay over the bottleneck link in each direction.

In Fig. 8, we plot the TCP congestion window size over time for each of the four flows. In this experiment, we chose CUBIC

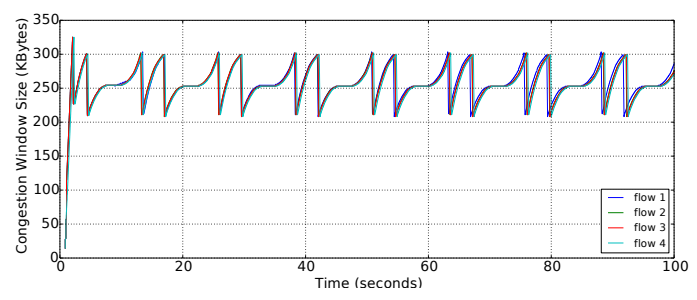

Figure 5: CPU limit $=0.01 * \mathrm{cpu}, \mathrm{TDF}=1.0$

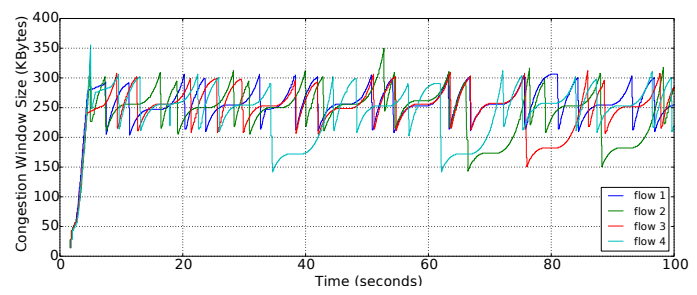

Figure 6: CPU limit $=0.005^{*} \mathrm{cpu}, \mathrm{TDF}=1.0$

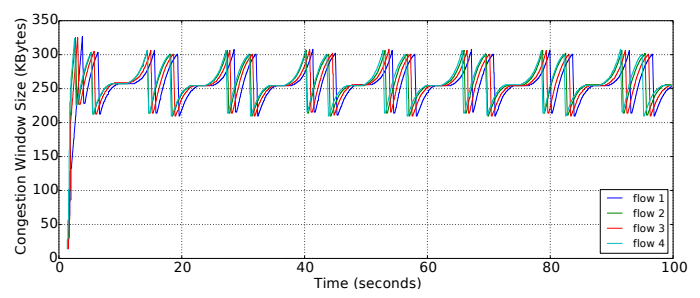

Figure 7: CPU limit $=0.005^{*} \mathrm{cpu}, \mathrm{TDF}=\mathbf{2 . 0}$

Figure 8: TCP congestion window size at different resource subscription levels.

as TCP congestion control algorithm. The results shown were obtained from three different scenarios. In the first scenario (top plot), we set the CPU limit to be $0.01 * c p u$ and TDF to be 1.0 for all ten containers; we use the results as the base for comparison. In the second scenario (middle plot), we reduced the CPU limit to $0.005 * c p u$ and maintained the same TDF. In this case, we have resource over-subscription. As a result, the plot clearly shows the introduced errors, which would be non-reproducible. In the third scenario (bottom plot), we both reduced the CPU limit to $0.005 * c p u$ and doubled TDF to 2.0. Using time dilation, our VTM system was able to compensate for the reduced resources and generated intended results.

\subsection{Different VM Scheduling}

In the third set of experiments, we aim to evaluate the system's ability to reproduce results under different scheduling order of the virtual machines and also with different time dilation. We simplified the dumbbell network in this case to have only four hosts connected by two routers, with links of specific bandwidth and delay, and generate two TCP flows 


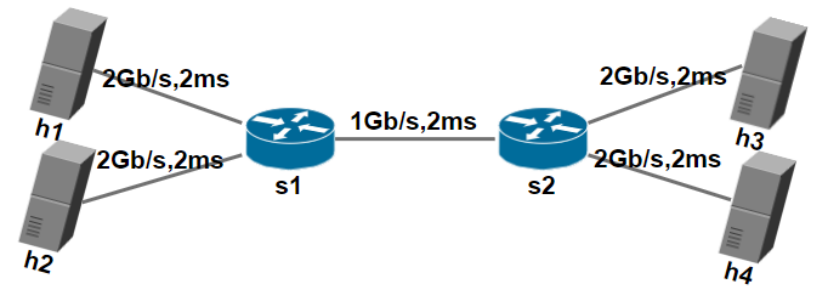

Figure 9: A dumbbell network setup with two flows.

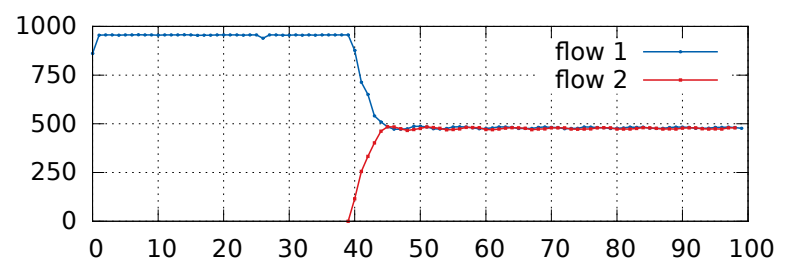

Figure 10: TCP throughput over time in three different scenarios (with different VM scheduling order and time dilation).

using iperf, as shown in Fig. 9. The two TCP flows interfered with each other over the $1 \mathrm{~Gb} / \mathrm{s}$ bottleneck link at the center of the dumbbell. We staggered the start time of the two flows: flow 1 started at time zero, and flow 2 started at around 40 seconds into the experiment.

Fig. 10 shows the throughput of the two flows over time. We ran three different scenarios. In the first scenario (top plot), the scheduling order was $\left(h_{1}, h_{2}, s_{1}, s_{2}, h_{3}, h_{4}\right)$, and the TDFs of all containers were set to be 2.0. In the second scenario, we changed the scheduling order to be $\left(s_{1}, h_{1}, s_{2}, h_{2}, h_{3}, h_{4}\right)$. In the third scenario, we maintained the same scheduling order as in the second case, but set the TDFs of the nodes differently: $\lambda_{h_{1}}=\lambda_{h_{2}}=1, \lambda_{s_{1}}=\lambda_{s_{2}}=2$, and $\lambda_{h_{3}}=\lambda_{h_{4}}=4$. The results from the three scenarios are similar.

Although the choice of the three scenarios seems to be arbitrary, the results provide good evidence that our VTM system is able to provide reproducible results independent of the scheduling ordering and time dilation setting of the virtual machines (as long as sufficient resources are available).

\section{CONCLUSION AND FUTURE WORK}

VTM is an integrated time dilation and VM scheduling mechanism for enabling reproducible network emulation experiments. VTM ensures precise time delivery of network messages in accordance with the bandwidth and delay specification of the target network. VTM is expected to reproduce results independent of the physical system capacity and the resource subscription level.

Results from preliminary experiments demonstrate the feasibility of the proposed method. In future work, we plan to conduct full system evaluation of large-scale network emulation experiments, including distributed emulation experiments. We plan to incorporate our system with Mininet and Maxinet [21] so that reproducibility will become a fully supported feature available to broader network researchers and scientists.

\section{ACKNOWLEDGMENT}

We thank the anonymous reviewers for their constructive comments. This research is supported in part by the National Natural Science Foundation of China under Project 61671086. This material is also based upon work supported in part by an NSF grant CNS-1563883 and a DHS grant 2017-ST-062000002 .

\section{REFERENCES}

[1] Association for Computing Machinery (ACM). 2019. Artifact Review and Badging. https://www.acm.org/publications/policies/ artifact-review-badging.

[2] Craig Bergstrom, Srinidhi Varadarajan, and Godmar Back. 2006. The Distributed Open Network Emulator: Using Relativistic Time for Distributed Scalable Simulation. In Proceedings of the 20th Workshop on Principles of Advanced and Distributed Simulation (PADS'O6). 19-28.

[3] Chameleon. 2019. https://www.chameleoncloud.org/.

[4] CloudLab. 2019. https://www.cloudlab.us/.

[5] Phillip M. Dickens, Philip Heidelberger, and David M. Nicol. 1996. Parallelized direct execution simulation of message-passing parallel programs. IEEE Transactions on Parallel and Distributed Systems 7, 10 (October 1996), 1090-1105.

[6] Miguel Erazo, Yue Li, and Jason Liu. 2009. SVEET! A scalable virtualized evaluation environment for TCP. In Proceedings of the 5th International Conference on Testbeds and Research Infrastructures for the Development of Networks and Communities (TridentCom'09).

[7] Global Environment for Network Innovations (GENI). 2019. http: //www.geni.net/.

[8] Andreas Grau, Steffen Maier, Klaus Herrmann, and Kurt Rothermel. 2008. Time Jails: A Hybrid Approach to Scalable Network Emulation. In Proceedings of the 22nd Workshop on Principles of Advanced and Distributed Simulation (PADS'O8). 7-14.

[9] Diwaker Gupta, Kashi Venkatesh Vishwanath, Marvin McNett, Amin Vahdat, Ken Yocum, Alex Snoeren, and Geoffrey M. Voelker. 2011. DieCast: Testing Distributed Systems with an Accurate Scale Model. ACM Trans. Comput. Syst. 29, 2, Article 4 (2011), 48 pages. https://doi.org/10.1145/1963559.1963560

[10] Diwaker Gupta, Ken Yocum, Marvin McNett, Alex C. Snoeren, Amin Vahdat, and Geoffrey M. Voelker. 2006. To Infinity and Beyond: Time-Warped Network Emulation. In Proceedings of the 3rd Symposium on Networked Systems Design and Implementation (NSDI'O6). 87-100.

[11] Nikhil Handigol, Brandon Heller, Vimalkumar Jeyakumar, Bob Lantz, and Nick McKeown. 2012. Reproducible Network Experiments Using Container-based Emulation. In Proc. of the 8th International Conference on Emerging Networking Experiments and Technologies (CoNEXT'12). 253-264.

[12] George Karypis and Vipin Kumar. 1998. A fast and high quality multilevel scheme for partitioning irregular graphs. SIAM J. Sci. Comput. 20 (1998), 359-392. Issue 1.

[13] Jereme Lamps, Vignesh Babu, David M. Nicol, Vladimir Adam, and Rakesh Kumar. 2018. Temporal Integration of Emulation and Network Simulators on Linux Multiprocessors. ACM Trans. Model. Comput. Simul. 28, 1, Article 1 (Jan. 2018), 25 pages.

[14] Jereme Lamps, David M. Nicol, and Matthew Caesar. 2014. TimeKeeper: A Lightweight Virtual Time System for Linux. In Proceedings of the 2nd ACM SIGSIM Conference on Principles of Advanced Discrete Simulation (SIGSIM-PADS'14). 179-186.

[15] Bob Lantz, Brandon Heller, and Nick McKeown. 2010. A Network in a Laptop: Rapid Prototyping for Software-defined Networks. In Proceedings of the 9th ACM SIGCOMM Workshop on Hot Topics in Networks (Hotnets-IX). Article 19, 6 pages.

[16] Jason Liu. 2008. A primer for real-time simulation of largescale networks. In Proceedings of the 41 st Annual Simulation Symposium (ANSS'08). 85-94.

[17] Jason Liu, Yougu Yuan, David M. Nicol, Robert S. Gray, Calvin C. Newport, David Kotz, and Luiz Felipe Perrone. 2004. Simulation 
Validation Using Direct Execution of Wireless Ad-Hoc Routing Protocols. In Proceedings of the 18th Workshop on Parallel and Distributed Simulation (PADS'04). 7-16.

[18] Xin Liu, Huaxia Xia, and Andrew A. Chien. 2003. Network emulation tools for modeling grid behavior. In Proceedings of $3 \mathrm{rd}$ IEEE/ACM International Symposium on Cluster Computing and the Grid (CCGrid'03).

[19] Nick McKeown, Tom Anderson, Hari Balakrishnan, Guru Parulkar, Larry Peterson, Jennifer Rexford, Scott Shenker, and Jonathan Turner. 2008. OpenFlow: Enabling Innovation in Campus Networks. SIGCOMM Comput. Commun. Rev. 38, 2 (March 2008), 69-74. https://doi.org/10.1145/1355734.1355746

[20] Open vSwitch. 2019. http://openvswitch.org/

[21] P. Wette et al. 2014. MaxiNet: Distributed emulation of softwaredefined networks. In IFIP Networking Conference. 1-9.

[22] Platforms for Advanced Wireless Research (PAWR). 2019. https: //www.advancedwireless.org/.

23] Steven K. Reinhardt, Mark D. Hill, James R. Larus, Alvin R. Lebeck, James C. Lewis, and David A. Wood. 1993. The Wisconsin Wind Tunnel: Virtual Prototyping of Parallel Computers. In Proceedings of the 1993 ACM SIGMETRICS Conference on Measurement and Modeling of Computer Systems. 48-60.
[24] Hajime Tazaki, Frédéric Uarbani, Emilio Mancini, Mathieu Lacage, Daniel Camara, Thierry Turletti, and Walid Dabbous. 2013. Direct Code Execution: Revisiting Library OS Architecture for Reproducible Network Experiments. In Proceedings of the 9th ACM Conference on Emerging Networking Experiments and Technologies (CoNEXT'13). 217-228.

[25] Elias Weingärtner, Florian Schmidt, Hendrik Vom Lehn, Tobias Heer, and Klaus Wehrle. 2011. SliceTime: A Platform for Scalable and Accurate Network Emulation. In Proceedings of the 8th USENIX Conference on Networked Systems Design and Implementation (NSDI'11). 253-266.

[26] Jiaqi Yan and Dong Jin. 2015. VT-Mininet: Virtual-time-enabled Mininet for Scalable and Accurate Software-Define Network Emulation. In Proceedings of the 1st ACM SIGCOMM Symposium on Software Defined Networking Research (SOSR'15). Article 27, 7 pages.

[27] Yuhao Zheng, Dong Jin, and David M. Nicol. 2013. Impacts of Application Lookahead on Distributed Network Emulation. In Proceedings of the 2013 Winter Simulation Conference (WSC'13). 2996-3007.

[28] Yuhao Zheng and David M. Nicol. 2011. A Virtual Time System for OpenVZ-Based Network Emulations. In Proceedings of the 2011 IEEE Workshop on Principles of Advanced and Distributed Simulation (PADS'11). 1-10. 